\title{
Comparative Value of Cholinesterase
}

\section{Inhibitors and Memantine in Persons with} Moderate-to-Severe Alzheimer's Disease in the United States: A Cost-Effectiveness

\section{Analysis $^{1}$}

\author{
Ismaeel Yunusa ${ }^{\mathrm{a}, *}$, Saud Alsahali ${ }^{\mathrm{b}}$, Amey Rane $^{\mathrm{c}}$ and Tewodros Eguale ${ }^{\mathrm{c}}$ \\ ${ }^{a}$ Center for Outcomes Research and Evaluation, Department of Clinical Pharmacy and Outcomes Sciences, \\ University of South Carolina College of Pharmacy, Columbia, SC, USA \\ ${ }^{\mathrm{b}}$ Department of Pharmacy Practice, Unaizah College of Pharmacy, Qassim University, Qassim, Saudi Arabia \\ ${ }^{\mathrm{c}}$ MCPHS University, Boston, MA, USA
}

Accepted 20 August 2021

Pre-press 31 August 2021

\begin{abstract}
.
Background: Pharmacological treatment of Alzheimer's disease (AD) involves symptomatic improvement of cognition using cholinesterase inhibitors (ChEIs) and memantine. The cost-effectiveness of these medications will guide decision-makers in making judicious use of scarce healthcare resources, particularly during the advanced disease stages.

Objective: To evaluate the cost-effectiveness of ChEIs, memantine, and ChEI-memantine combinations in persons with moderate-to-severe AD from the US healthcare perspective.

Methods: This pharmacoeconomic evaluation study used a state-transition Markov cohort model to simulate the costs and effectiveness of ChEI-memantine combinations compared with monotherapies of ChEI (donepezil, galantamine and rivastigmine) and memantine in persons with moderate-to-severe $\mathrm{AD}$ over a lifetime horizon with a 1-year cycle length. We estimated expected quality-adjusted life-years (QALYs), costs (in 2020 \$US), net monetary benefits, and incremental cost-effectiveness ratios (ICERs). We discounted future costs and QALYs at the rate of 3\%.

Results: In this study, donepezil monotherapy, galantamine-memantine combination, and rivastigmine transdermal patch formed the cost-effectiveness frontier. Findings suggests that rivastigmine transdermal patch is the optimal treatment strategy at a willingness-to-pay (WTP) threshold of $\$ 150,000 /$ QALY (ICER $=\$ 93,307 /$ QALY [versus donepezil monotherapy]). Results across subgroups by age and sex also suggest that the rivastigmine transdermal patch is the optimal treatment strategy with the highest net benefit.

Conclusion: From the US healthcare perspective, we found that, for persons with moderate-to-severe AD at a WTP threshold of $\$ 150,000 / \mathrm{QALY}$, the rivastigmine transdermal patch is the most cost-effective pharmacological treatment option. Given that the transdermal patch is a preferred route of administration for persons with $\mathrm{AD}$ and their caregivers due to its convenience, our findings provide additional incentives for its use.
\end{abstract}

Keywords: Alzheimer's disease, cholinesterase inhibitors, cost-effectiveness analysis, donepezil, memantine, quality-adjusted life years, rivastigmine

\footnotetext{
${ }^{1}$ This article received a correction notice (Erratum) with the reference: 10.3233/ADR-219005, available at https://content.ios press.com/articles/journal-of-alzheimers-disease-reports/adr2190 05.
}

${ }^{*}$ Correspondence to: Ismaeel Yunusa, PharmD, PhD, Center for Outcomes Research and Evaluation, Department of Clinical Pharmacy and Outcomes Sciences, University of South Carolina College of Pharmacy, Columbia, SC, USA. E-mail: Iyunusa@mailbox.sc.edu. 


\section{INTRODUCTION}

The number of persons with Alzheimer's disease (AD) in the United States (US) is rising as the population of older adults increases [1]. AD is a growing concern due to longer life expectancy and the considerable size of the population expected to reach age 65 and beyond in the coming decades. An estimated 6.2 million people aged 65 and older in the US are living with $\mathrm{AD}[2,3]$. It is the most common type of dementia and accounts for $60-80 \%$ of reported cases [4]. Among individuals in the US aged 65 years or older, more than 1 in 9 has $\mathrm{AD}[2,5]$. AD is the fifth leading cause of death in persons aged 65 years or older [6]. In 2018, the US society spent an estimated $\$ 277$ billion in the cost of care of persons with AD and other dementias [3]. Currently, AD has no cure, and pharmacological options for the management of its symptom are limited.

Pharmacological treatment for $\mathrm{AD}$ comprises memantine and the cholinesterase inhibitors (ChEIs) donepezil, galantamine, and rivastigmine. Although most cases progress to a severe stage that can last many years, most published clinical and economic evaluations have focused on the mild-to-moderate stages [7, 8]. The American Academy of Family Physicians recommends that treatment for mildto-moderate AD should begin with ChEIs [9]. Donepezil, galantamine, and rivastigmine are FDAapproved for use during these stages. Switching ChEIs for lack of response or intolerable adverse effects is acceptable. For moderate-to-severe AD, studies suggest that combining memantine with an ChEI might help [10, 11]. Donepezil and the rivastigmine transdermal patch are FDA-approved for all stages, and memantine and donepezil-memantine combination for moderate-to-severe AD.

About $75 \%$ of the healthcare costs associated with $\mathrm{AD}$ occur during the more severe stages [12]. Therefore, the cost-effectiveness of medications during later stages is important to guide decision-making for judicious use of scarce healthcare resources. Previous cost-effectiveness studies of pharmacological treatments of moderate-to-severe AD have not compared all relevant drug combinations in a single decision analytic model (e.g., only comparing donepezil-memantine versus donepezil monotherapy [13], or memantine with ChEI as a drug class versus ChEI-class monotherapies; [14] both based on single clinical trials). It is unknown which ChEImemantine combination is most cost-effective, since combinations differ in cost and effectiveness [14, 15].
We aimed to conduct a cost-effectiveness analysis of treating moderate-to-severe $\mathrm{AD}$ with all the clinically plausible and relevant treatment alternatives; the combination of memantine with each ChEI versus best supportive care (BSC) or ChEI monotherapy or memantine monotherapy.

\section{METHODS}

\section{Model description and treatment strategies}

This report follows the Consolidated Health Economic Evaluation Reporting Standards (CHEERS) guideline for health economic evaluations [16, 17]. Following guidance from the US Panel on Costeffectiveness in Health and Medicine [18, 19], we developed a state-transition Markov model [20] to evaluate the cost-effectiveness of the following nine strategies in treating moderate-to-severe AD: BSC; donepezil or galantamine or oral rivastigmine or rivastigmine transdermal patch or memantine monotherapy; donepezil-memantine, galantaminememantine or oral rivastigmine-memantine combinations.

The model begins with an initial distribution of patients in moderate and severe health states and recursively applies annual transition probabilities to simulate how patients switch states (to or from mild, moderate, or severe $\mathrm{AD}$, or to death from $\mathrm{AD}$ or death from other causes; Fig. 1). Death from other causes was estimated from the US Life Table (Supplementary Table 1), stratified by sex (Supplementary Table 2) [21].

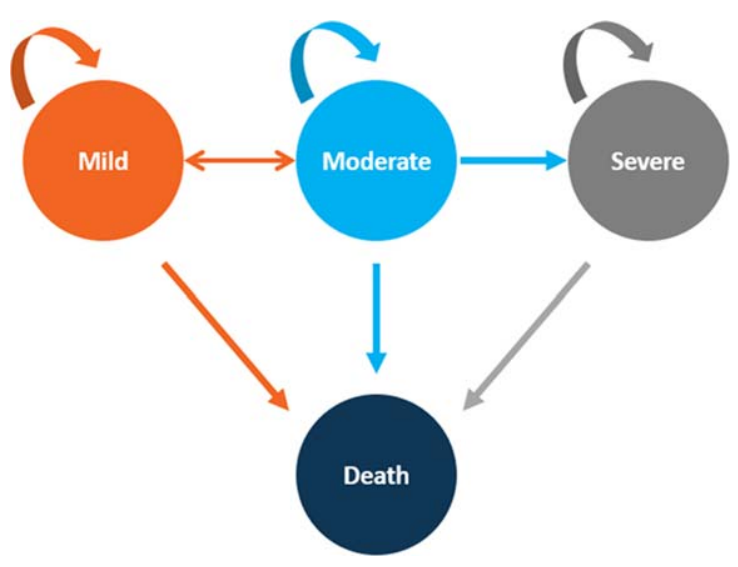

Fig. 1. Health-State Transition Diagram. Depicts the Markov model health state transition. The circles represent health states, and the arrows represent possible transitions from one health state to another. 


\section{Model parameters}

\section{Transition probabilities}

We obtained data for patients aged $\geq 65$ with AD enrolled in approximately 29 Alzheimer's Disease Centers (ADCs) between 2005-2015 from the National Alzheimer's Coordinating Center (NACC) database. ADCs are funded by the National Institute on Aging (NIA) of the National Institutes of Health $[22,23]$. The ADCs enroll and follow participants approximately annually and NACC provides data for research upon request; all participants provided written informed consent. An Institutional Review Board (IRB) at each ADC approved all research activities, including data sharing; this secondary analysis of the de-identified data did not require additional approval.

Selected patients had $\geq 1$ annual visit to the ADCs. Health states were determined using validated Clinical Dementia Rating Scale $\left(\mathrm{CDR}^{\circledR}\right.$ Dementia Staging Instrument) scores [24]: CDR $0.5-1$ is mild; 2 is moderate and 3 is severe $[25,26]$. These categories correlate with other AD measures [24]. We used the msm package in $\mathrm{R}$ to develop a multistate model $[27,28]$ and calculate the annual transition probabilities for treated or untreated patients aged 65 years. The disease stages were modelled as homogenous continuous-time Markov process with a transition $\mathrm{Q}$ matrix [28]. The calculated transition probabilities were consistent with others using the same database from a shorter timeframe (2005-2009) [29]. These probabilities were used to populate the transition matrix (Supplementary Table 3).

\section{Intervention effectiveness and model assumptions}

We incorporated the effect of interventions from a Bayesian network meta-analysis (NMA) of 142 studies [15]. This provides the most comprehensive and current evidence of the effectiveness of pharmacological treatments of $\mathrm{AD}$, including indirect treatment estimates. The effect of the drugs on mortality (versus BSC) are presented in Supplementary Table 4. We converted the odds ratio (OR) to relative risk (RR) using the formula by Zhang et al. [30] before incorporating treatment effects for pertinent transition probabilities into the model using the RRs.

Although this NMA provided direct and indirect evidence for most of the strategies, we were unable to obtain data on transitioning from moderate or severe to death for rivastigmine-memantine, so we assumed the transition probability to be identical to donepezil- memantine because the drugs are similar, and this is the most studied combination in clinical trials with the most robust evidence.

\section{Costs and utilities}

All cost inputs were inflation-adjusted to 2020 US dollar value using the Consumer Price Index from the US Bureau of Labor Statistics. We included direct medical, non-medical, and caregiving costs. Cost of drugs acquisition were obtained from the 2016 Medicare Provider Utilization and Payment Data from the US Centers for Medicare \& Medicaid Services (CMS) [31], except for rivastigmine transdermal patch which was unavailable in CMS so was obtained from the Average Wholesale Price (AWP) using the RED BOOK (Truven Health Analytics) [32]. We derived cost of care and utility estimates by disease stage based on an analysis by SaintLaurent Thibault et al. (2015) [14] (Supplementary Table 5). We used direct medical cost of care (including antipsychotic medications, monitoring, physician visits and home care) to reflect the healthcare payer perspective (typically reimbursed by Medicare) [14].

\section{Model outcomes and analysis}

We defined outcomes (QALYs and costs) from the model over a lifetime horizon (i.e., the patient's lifetime which was until every patient dies, and consistent with studies that have indicated that people $\geq 65$ can live between 4 to 20 years) [3]. We assessed cost-effectiveness for each strategy by estimating the expected ICERs and net monetary benefits (NMBs). Expected ICER was estimated as the difference in cost between treatments divided by the difference in effect. In this cost-effectiveness analysis, comparison was made in the following ways [33]. 1) We arranged alternatives in order of increasing effectiveness. 2) We ruled out strongly dominated alternatives. 3) We calculated ICERs based on moving to increasingly costly and increasingly effective alternatives. 4) If the ICER associated with moving to more costly alternative falls, then the lower-cost alternative used to calculate the ICER is extendedly dominated and was ruled out. 5) We then recalculated ICERs based on comparisons of moving to increasingly costly but increasingly effective alternatives that are neither strongly nor extendedly dominated. Expected NMB was estimated as the effectiveness (in QALYs) multiplied by the willingness-to-pay (WTP) threshold, minus the cost of treatments. Using the NMB, which is found to be consistent with ICER approach, treat- 
ment with the greatest net benefit was determined as the most optimal treatment [33].

We applied a $3 \%$ discounting rate for costs and outcomes as recommended by the US Panel on Cost-effectiveness in Health and Medicine [18, 19]. A WTP threshold of $\$ 100,000$ or $\$ 150,000$ per QALY has been recommended (outside the context of an explicit resource constraint) [34]. We used $\$ 150,000 /$ QALY to determine the optimal strategy in the reference-case and sensitivity analyses. Decisionmakers may use the WTP threshold of their choice within the context of an explicit resource constraint.

To account for heterogeneity within the model, we conducted subgroup analyses on males, female, and starting age of 74 years, since age and sex are among the most important risk factors for AD, for which outcomes might differ [3]. All modelling computations were conducted using hēRo3, a web-based, open-source health economic modeling platform (https://heroapps.io/).

\section{Sensitivity analysis}

Deterministic, one-way sensitivity analysis was conducted by varying some selected variables through plausible ranges to assess the robustness of our results. Specifically, we varied the cost of rivastigmine transdermal patch, given that costs were from the RED BOOK while other drug costs were from CMS. We also varied the probabilities of moving to death from moderate and severe health states for rivastigmine-memantine as the probability we used was assumed to equal that of donepezil-memantine due to lack of data. This one-way analysis varied the estimates by $\pm 25 \%$ of the base case values, consistent with the literature where ranges are unavailable [35].
To account for joint parameter uncertainty in the entire model, we conducted a probabilistic sensitivity analysis (PSA) using second-order Monte Carlo simulations [36] based on 1000 iterations using distributions appropriate to the parameters: beta distribution for transition probabilities (adequately reflects a binomial distribution for a fraction of event and no event; bounded between 0 and 1 ), normal distributions for costs (data from a large sample size [37, 38]), and lognormal distribution for utilities (suitable when health states close to death are possible, such as severe $\mathrm{AD}[37,38])$.

\section{RESULTS}

Table 1 presents the results for all nine strategies. Donepezil, galantamine-memantine combination, and rivastigmine transdermal patch formed the cost-effectiveness or efficiency frontier (Fig. 2) [39]. In the reference-case analysis, the most effective treatment is rivastigmine transdermal patch (2.250 QALYs), followed by rivastigminememantine (1.831 QALYs). BSC and oral rivastigmine are the least effective ( 0.755 and 0.876 QALYs, respectively). Apart from rivastigmine transdermal patch, all combination treatments were more effective than their monotherapies. Subgroup analyses suggest that treatments are more effective in females than males and at 65 than 74 years (Tables 1 and 2).

The reference-case result indicates that rivastigmine oral and galantamine monotherapy were strongly dominated by galantamine-memantine as they cost more and were less effective (Fig. 2 and Supplementary Figure 1). Memantine was dominated by donepezil and rivastigmine-memantine was extendedly dominated by rivastigmine transdermal patch.

Table 1

Reference-Case Cost-Effectiveness of Cholinesterase Inhibitors and Memantine in Moderate-to-Severe Alzheimer's Disease

\begin{tabular}{lcclcc}
\hline Intervention & QALYs & Cost, \$ & ICER, \$/QALY & NMB, \$ \\
\hline Best supportive care & 0.755 & 38,473 & Strongly dominated (versus galantamine-memantine) & 74,777 \\
Rivastigmine oral monotherapy & 0.876 & 58,277 & Strongly dominated (versus donepezil monotherapy) & 73,123 \\
Galantamine monotherapy & 0.908 & 60,793 & Strongly dominated (versus donepezil monotherapy) & 75,407 \\
Galantamine-memantine & 0.961 & 37,335 & - & & 106,815 \\
Memantine monotherapy & 1.294 & 48,728 & Strongly dominated (versus donepezil monotherapy) & 145,372 \\
Donepezil monotherapy & 1.609 & 48,176 & 16,739 (versus galantamine-memantine) & 193,174 \\
Donepezil-memantine & 1.795 & 73,423 & Extendedly dominated (versus rivastigmine transdermal patch) & 195,827 \\
Rivastigmine-memantine & 1.831 & 89,832 & Extendedly dominated (versus rivastigmine transdermal patch) & 184,818 \\
Rivastigmine transdermal patch & 2.250 & 108,036 & 93,307 & 229,464 \\
\hline
\end{tabular}

QALYs, quality-adjusted life-years; ICER, incremental cost-effectiveness ratio (expressed in 2020 US dollars per QALY); Strongly dominated, means that a treatment is ruled out because it costs more for fewer QALYs than the comparator alternative; extendedly dominated means that a treatment is ruled out because it has an ICER that is greater than that of a more effective comparator alternative. 


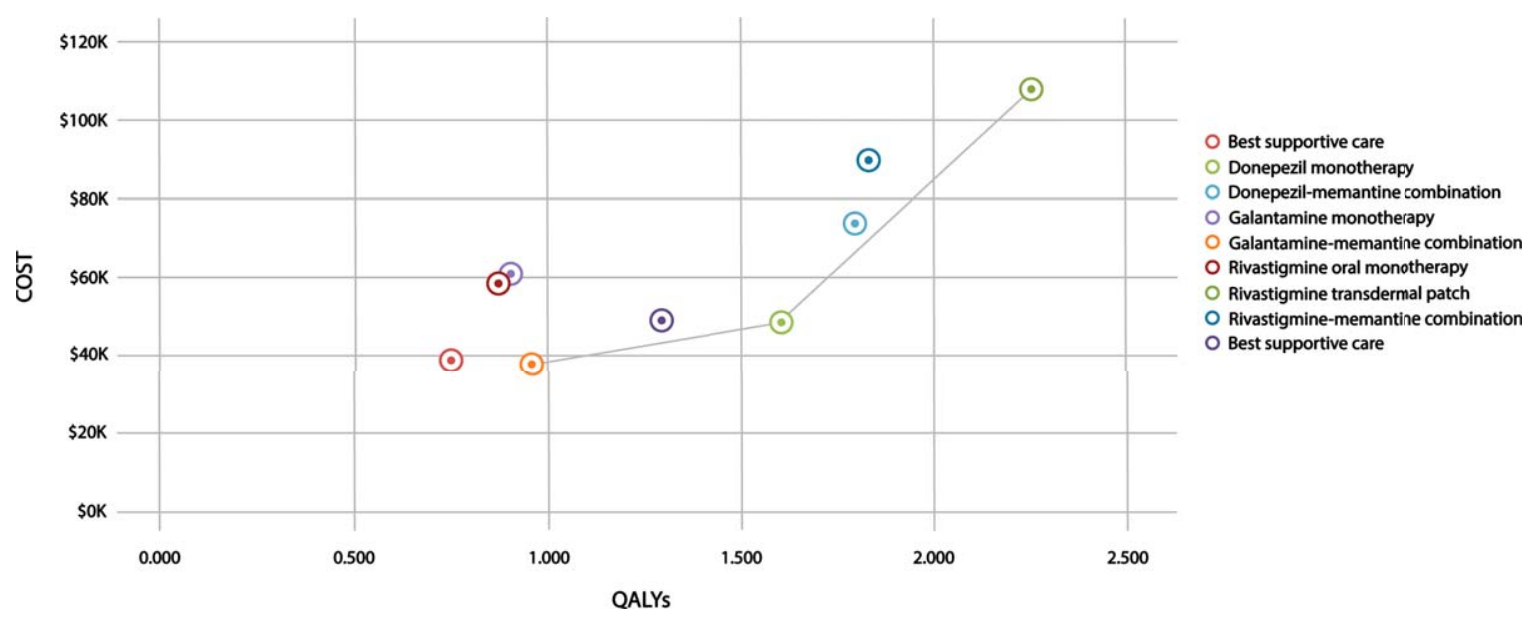

Fig. 2. Cost-effectiveness plane. Shows the cost-effectiveness plane for costs and outcomes (quality-adjusted life-years) associated with all possible treatment strategies for AD. The solid line represents the cost-effective frontier.

Table 2

Cost-Effectiveness of Cholinesterase Inhibitors and Memantine Among Age and Sex Subgroups

\begin{tabular}{|c|c|c|c|c|c|c|c|c|c|}
\hline \multirow[b]{2}{*}{ Intervention } & \multicolumn{3}{|c|}{ Male } & \multicolumn{3}{|c|}{ Female } & \multicolumn{3}{|c|}{ Age 74} \\
\hline & QALYs & Cost, \$ & $\mathrm{NMB}, \$^{\mathrm{a}}$ & $\overline{\text { QALYs }}$ & Cost, \$ & $\mathrm{NMB}, \$^{\mathrm{a}}$ & $\overline{\text { QALYs }}$ & Cost, \$ & $\mathrm{NMB}, \$^{\mathrm{a}}$ \\
\hline Best supportive care & 0.747 & 37,992 & 74,058 & 0.759 & 38,731 & 75,119 & 0.726 & 36,560 & 72,340 \\
\hline Rivastigmine oral monotherapy & 0.866 & 57,400 & 72,500 & 0.882 & 58,750 & 73,550 & 0.834 & 54,702 & 70,398 \\
\hline Galantamine monotherapy & 0.896 & 59,596 & 74,804 & 0.915 & 61,437 & 75,813 & 0.859 & 55,774 & 73,076 \\
\hline Galantamine-memantine & 0.953 & 37,020 & 105,930 & 0.965 & 37,505 & 107,245 & 0.932 & 36,138 & 103,662 \\
\hline Memantine monotherapy & 1.279 & 48,138 & 143,712 & 1.302 & 49,046 & 146,254 & 1.235 & 46,386 & 138,864 \\
\hline Donepezil monotherapy & 1.584 & 47,389 & 190,211 & 1.622 & 48,600 & 194,700 & 1.507 & 44,940 & 181,110 \\
\hline Donepezil-memantine & 1.763 & 71,999 & 192,451 & 1.813 & 74,190 & 197,760 & 1.662 & 67,458 & 181,842 \\
\hline Rivastigmine-memantine & 1.798 & 88,092 & 181,608 & 1.849 & 90,770 & 186,580 & 1.693 & 82,541 & 171,409 \\
\hline Rivastigmine transdermal patch & 2.198 & 105,341 & 224,359 & 2.278 & 109,486 & 232,214 & 2.027 & 96,440 & 207,610 \\
\hline
\end{tabular}

QALY, quality-adjusted life-years; ICER, Incremental cost-effectiveness ratio (expressed in 2020 US dollars per QALY); NMB, Net monetary benefit. ${ }^{a}$ Net monetary benefit at willingness-to-pay threshold of \$150,000/QALY. Treatments with the greatest net monetary benefits are highlighted in bold.

This cost-effectiveness analysis suggests that rivastigmine transdermal patch is the optimal treatment strategy at a WTP value of $\$ 150,000 / \mathrm{QALY}$ (ICER $=\$ 93,307 /$ QALY versus donepezil monotherapy), with similar results across subgroups.

One-way sensitivity analysis by varying the cost of rivastigmine transdermal patch and probabilities of rivastigmine-memantine combination from moderate or severe $\mathrm{AD}$ to death did not change suggestions on the optimal treatment (ICER $<$ WTP) as both variables did not remarkably influence the ICER.

The PSA result based on healthcare perspective at WTP value of $\$ 150,000 / \mathrm{Q} A L Y$ suggests that rivastigmine transdermal patch was the optimal treatment in $78.2 \%$ of iterations followed by donepezil-memantine $(11.6 \%)$; then rivastigmine-memantine $(7.9 \%)$; and then donepezil monotherapy (2.3\%) (Fig. 3).

\section{DISCUSSION}

In this study for patients with moderate-to-severe $\mathrm{AD}$, we examined the cost-effectiveness of ChEImemantine combinations versus memantine or ChEI monotherapy. Based on the US healthcare perspective, our results suggest that monotherapy with the rivastigmine transdermal patch is the optimal treatment strategy at a WTP value of $\$ 150,000$ per QALY. These results were consistent across subgroups. Combination therapies were more effective (provided more QALYs) than their respective monotherapies, except for the rivastigmine transdermal patch.

Previous cost-effectiveness studies on treatments for moderate-to-severe $\mathrm{AD}$, which did not compare all available treatment options and used a shorter 


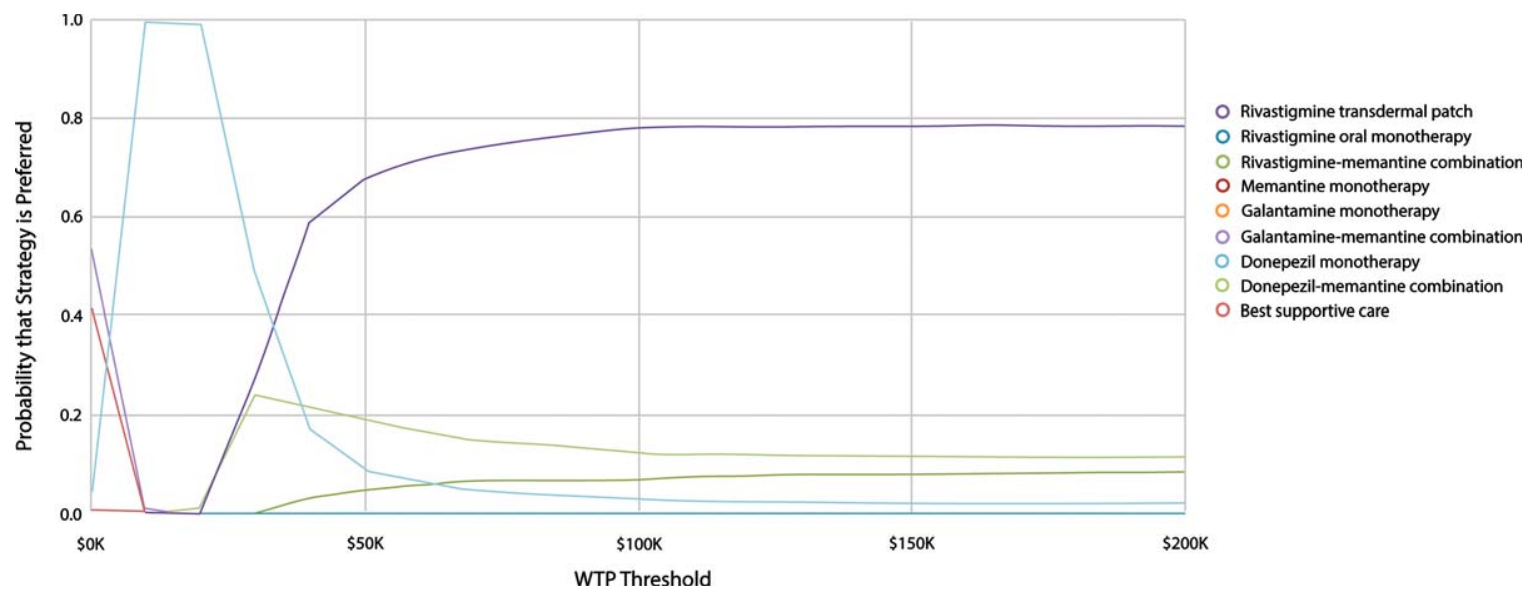

Fig. 3. Probabilistic Sensitivity Analysis Cost-Effectiveness Acceptability Curve. Shows Cost-Effectiveness Acceptability Curves for nine different treatment options. The vertical axis represents probability that a treatment option is cost-effective while the horizontal axis presents the willingness-to-pay (WTP) threshold range (i.e., $\$ 0-\$ 200,000$ per QALY). Rivastigmine transdermal patch has the highest probability to be cost-effective ("optimal") at WTP values $\geq \$ 50,000$ per QALY.

time horizon, yielded results that differed from ours. Weycker et al. (2007) [13] compared memantinedonepezil versus donepezil monotherapy, for only a year time horizon, and concluded that the combination was more cost-effective. Saint-Laurent Thibault et al. (2015) [14] compared ChEI-memantine versus memantine or ChEIs monotherapy over a 3-year time horizon and found that the combination was cost-effective. However, the study did not include the rivastigmine transdermal patch and did not differentiate between the three possible ChEI combinations.

The estimates of drug effect in both these studies were based on single clinical trials.

In the UK, a cost-effectiveness analysis by Knapp et al. (2017) with model inputs derived from a pragmatic clinical trial compared no treatment, donepezil monotherapy, memantine monotherapy, and donepezil-memantine [40-42]. It found that donepezil-memantine was not more cost-effective than donepezil monotherapy. It called for the UK National Institute for Health and Care Excellence (NICE) to revise its reimbursement guidelines [40, 43], which were based on the conclusion that insufficient evidence exists to differentiate between ChEIs. The NICE guidelines recommended that the best use of resources would be the option with the lowest acquisition cost [43]. However, in economic evaluations, decisions on optimal treatments should be based on maximizing net benefits rather than differences in statistical significance, given that the magnitude of costs and effects are not the same [44].
Failing to include all relevant alternatives could result in overestimation of cost-effectiveness if an intervention was not compared with a more cost-effective treatment [33]. Including all possible alternatives can be challenging because randomized controlled trials (RCTs) rarely compare all relevant treatment strategies, and we were able to use the NMA of 142 studies (published after the above three previous studies) to include treatments that were never compared directly in clinical trials [15]. To be able to completely assess the cost-effectiveness of interventions, it is also necessary that the time horizon should be long enough to capture all relevant outcomes [33], and our estimate is based on a lifetime horizon.

Since the introduction of tacrine in 1993 (withdrawn from the US market in 2012), and until new treatments are developed, memantine and the three ChEIs remain the mainstay in the symptomatic treatment of AD. It is worth noting, however, that in May 2018, the French Ministry of Health announced a policy that removed all AD drugs (donepezil, galantamine, rivastigmine, and memantine) from its list of reimbursable treatments [45]. If our US costeffectiveness findings also apply to France, this 2018 policy decision in effect denies treatment that delivers more than two QALYs.

The high probability of being cost-effective we found for rivastigmine transdermal patch (78\% from the healthcare perspective) gives an insight into the level of parameter uncertainty and supports our conclusion. This is one example of the value of our 
cost-effectiveness analysis, being able to guide decision makers regarding the best use of resources among available treatment options.

\section{Strength and limitations}

To our knowledge, our study is the first costeffectiveness analysis of ChEI-memantine combination therapy versus ChEI or memantine monotherapy in the treatment of moderate-to-severe AD that compares all relevant alternatives, making available the most complete set of data for decision makers. In addition, our study accounted for model heterogeneity to evaluate differences in results due to sex or age.

As with any simulation model, our study should be viewed in the context of some limitations. First, our cohort consist of patients with a starting age of 65 (and a subgroup of 74) years; it is not clear whether our conclusion is applicable to other starting ages. This limitation is significant, because older patients have a higher mortality rate, and they are at higher risk of disease progression than younger ones. In the future, it will be important to evaluate the costeffectiveness of these agents at other ages. Another limitation is that the model in the current study did not include differences in side effects of drugs. However, clinical experts indicate that, over long-term use, these medications are equally tolerated. Moreover, we accounted for adverse effects and drug switching in the MSM used to generate transition probabilities. Although a small proportion of NACC participants are in a skilled nursing facility, nursing home, hospital, or hospice, this study was limited to majority of patients in the community, as costs and utility inputs of the model were based on those reported for patients in such settings. Institutionalized patients incur more healthcare cost, and hence it is unclear whether our findings regarding optimal treatment would be applicable to these patients.

\section{CONCLUSION}

From the US healthcare perspective, we found that, for moderate-to-severe $\mathrm{AD}$ patients aged 65 years, at a WTP value of $\$ 150,000$, the rivastigmine transdermal patch was the most cost-effective treatment. On examining parameter uncertainty, rivastigmine also had the greatest probability of being cost-effective. Given that the transdermal patch is a preferred route of administration for $\mathrm{AD}$ patients and caregivers due to its convenience, our findings provide an additional incentive for its use. These finding are consistent across subgroups by age and sex. Future studies may explore the cost-effectiveness of these agents in other age groups and institutional settings.

\section{ACKNOWLEDGMENTS}

Data used to estimate transition probabilities that went into the Model in this study was from the NACC database which is funded by NIA/NIH Grant U01 AG016976. NACC data are contributed by the NIA funded ADCs: P30 AG019610 (PI Eric Reiman, MD), P30 AG013846 (PI Neil Kowall, MD), P30 AG062428-01 (PI James Leverenz, MD) P50 AG 008702 (PI Scott Small, MD), P50 AG025688 (PI Allan Levey, MD, PhD), P50 AG047266 (PI Todd Golde, MD, PhD), P30 AG010133 (PI Andrew Saykin, PsyD), P50 AG005146 (PI Marilyn Albert, PhD), P30 AG062421-01 (PI Bradley Hyman, MD, PhD), P30 AG062422-01 (PI Ronald Petersen, MD, PhD), P50AG005138 (PI Mary Sano, PhD), P30 AG008051 (PI Thomas Wisniewski, MD), P30 AG 013854 (PI Robert Vassar, PhD), P30 AG008017 (PI Jeffrey Kaye, MD), P30 AG010161 (PI David Bennett, MD), P50 AG047366 (PI Victor Henderson, MD, MS), P30 AG010129 (PI Charles DeCarli, MD), P50 AG016573 (PI Frank LaFerla, PhD), P30 AG062429-01(PI James Brewer, MD, PhD), P50 AG023501 (PI Bruce Miller, MD), P30 AG035982 (PI Russell Swerdlow, MD), P30 AG028383 (PI Linda Van Eldik, PhD), P30 AG053760 (PI Henry Paulson, MD, PhD), P30 AG010124 (PI John Trojanowski, MD, PhD), P50 AG005133 (PI Oscar Lopez, MD), P50 AG005142 (PI Helena Chui, MD), P30 AG012300 (PI Roger Rosenberg, MD), P30 AG049638 (PI Suzanne Craft, PhD), P50 AG005136 (PI Thomas Grabowski, MD), P30 AG062715-01 (PI Sanjay Asthana, MD, FRCP), P50 AG005681 (PI John Morris, MD), P50 AG047270 (PI Stephen Strittmatter, MD, PhD).

\section{CONFLICT OF INTEREST}

The authors have no conflict of interest to report.

\section{SUPPLEMENTARY MATERIAL}

The supplementary material is available in the electronic version of this article: https://dx.doi.org/ 10.3233/ADR-210307. 


\section{REFERENCES}

[1] Reisberg B, Doody R, Stoffler A, Schmitt F, Ferris S, Mobius HJ (2003) Memantine in moderate-to-severe Alzheimer's disease. N Engl J Med 348, 1333-1341.

[2] (2021) 2021 Alzheimer's disease facts and figures. Alzheimers Dement 17, 327-406.

[3] (2018) 2018 Alzheimer's disease facts and figures. Alzheimers Dement 14, 367-429.

[4] (2014) 2014 Alzheimer's disease facts and figures. Alzheimers Dement 10, e47-e92.

[5] (2015) 2015 Alzheimer's disease facts and figures. Alzheimers Dement 11, 332-384.

[6] (2017) 2017 Alzheimer's disease facts and figures. Alzheimers Dement 13, 325-373.

[7] Cappell J, Herrmann N, Cornish S, Lanctôt KL (2010) The pharmacoeconomics of cognitive enhancers in moderate to severe Alzheimer's disease. CNS Drugs 24, 909-927.

[8] Davis M, O Connell T, Johnson S, Cline S, Merikle E, Martenyi F, Simpson K (2018) Estimating Alzheimer's disease progression rates from normal cognition through mild cognitive impairment and stages of dementia. Curr Alzheimer Res 15, 777-788.

[9] Qaseem A, Snow V, Cross JT Jr, Forciea MA, Hopkins R Jr, Shekelle P, Adelman A, Mehr D, Schellhase K, CamposOutcalt D, Santaguida P, Owens DK; American College of Physicians/American Academy of Family Physicians Panel on Dementia (2008) Current pharmacologic treatment of dementia: A clinical practice guideline from the American College of Physicians and the American Academy of Family Physicians. Ann Intern Med 148, 370-378.

[10] Parsons CG, Danysz W, Dekundy A, Pulte I (2013) Memantine and cholinesterase inhibitors: Complementary mechanisms in the treatment of Alzheimer's disease. $\mathrm{Neu}$ rotox Res $\mathbf{2 4}, 358-369$.

[11] Farrimond LE, Roberts E, McShane R (2012) Memantine and cholinesterase inhibitor combination therapy for Alzheimer's disease: A systematic review. BMJ Open 2, e000917.

[12] Wimo A, Winblad B, Stoffler A, Wirth Y, Mobius HJ (2003) Resource utilisation and cost analysis of memantine in patients with moderate to severe Alzheimer's disease. Pharmacoeconomics 21, 327-340.

[13] Weycker D, Taneja C, Edelsberg J, Erder MH, Schmitt FA, Setyawan J, Oster G (2007) Cost-effectiveness of memantine in moderate-to-severe Alzheimer's disease patients receiving donepezil. Curr Med Res Opin 23, 1187-1197.

[14] Saint-Laurent Thibault C, Ozer Stillman I, Chen S, Getsios D, Proskorovsky I, Hernandez L, Dixit S (2015) Cost-utility analysis of memantine extended release added to cholinesterase inhibitors compared to cholinesterase inhibitor monotherapy for the treatment of moderate-tosevere dementia of the Alzheimer's type in the US. J Med Econ 18, 930-943.

[15] Tricco AC, Ashoor HM, Soobiah C, Rios P, Veroniki AA, Hamid JS, Ivory JD, Khan PA, Yazdi F, Ghassemi M, Blondal E, Ho JM, Ng CH, Hemmelgarn B, Majumdar SR, Perrier L, Straus SE (2018) Comparative effectiveness and safety of cognitive enhancers for treating Alzheimer's disease: Systematic review and network metaanalysis. $J \mathrm{Am}$ Geriatr Soc 66, 170-178.

[16] Husereau D, Drummond M, Petrou S, Carswell C, Moher D, Greenberg D, Augustovski F, Briggs AH, Mauskopf
J, Loder E (2013) Consolidated Health Economic Evaluation Reporting Standards (CHEERS) statement. BMJ 346, f1049.

[17] Husereau D, Drummond M, Petrou S, Carswell C, Moher D, Greenberg D, Augustovski F, Briggs AH, Mauskopf J, Loder E; ISPOR Health Economic Evaluation Publication Guidelines-CHEERS Good Reporting Practices Task Force (2013) Consolidated Health Economic Evaluation Reporting Standards (CHEERS)-Explanation and elaboration: A report of the ISPOR Health Economic Evaluation Publication Guidelines Good Reporting Practices Task Force. Value in Health 16, 231-250.

[18] Sanders GD, Neumann PJ, Basu A, Brock DW, Feeny D, Krahn M, Kuntz KM, Meltzer DO, Owens DK, Prosser LA, Salomon JA, Sculpher MJ, Trikalinos TA, Russell LB, Siegel JE, Ganiats TG (2016) Recommendations for Conduct, Methodological Practices, and Reporting of Costeffectiveness Analyses: Second Panel on Cost-Effectiveness in Health and Medicine. JAMA 316, 1093-1103.

[19] Neumann PJ, Sanders GD, Russell LB, Siegel JE, Ganiats TG (2016) Cost-Effectiveness in Health and Medicine, Oxford University Press.

[20] Siebert U, Alagoz O, Bayoumi AM, Jahn B, Owens DK, Cohen DJ, Kuntz KM (2012) State-transition modeling: A report of the ISPOR-SMDM Modeling Good Research Practices Task Force-3. Value Health 15, 812-820.

[21] Arias E, Xu J (2018) United States Life Tables, 2015. Natl Vital Stat Rep 67, 1-64.

[22] Weintraub S, Salmon D, Mercaldo N, Ferris S, GraffRadford NR, Chui H, Cummings J, DeCarli C, Foster NL, Galasko D, Peskind E, Dietrich W, Beekly DL, Kukull WA, Morris JC (2009) The Alzheimer's Disease Centers' Uniform Data Set (UDS): The neuropsychologic test battery. Alzheimer Dis Assoc Disord 23, 91-101.

[23] Morris JC, Weintraub S, Chui HC, Cummings J, Decarli C, Ferris S, Foster NL, Galasko D, Graff-Radford N, Peskind ER, Beekly D, Ramos EM, Kukull WA (2006) The Uniform Data Set (UDS): Clinical and cognitive variables and descriptive data from Alzheimer Disease Centers. Alzheimer Dis Assoc Disord 20, 210-216.

[24] Hughes CP, Berg L, Danziger WL, Coben LA, Martin RL (1982) A new clinical scale for the staging of dementia. $\mathrm{Br}$ J Psychiatry 140, 566-572.

[25] Juva K, Sulkava R, Erkinjuntti T, Ylikoski R, Valvanne J, Tilvis R (1994) Staging the severity of dementia: Comparison of clinical (CDR, DSM-III-R), functional (ADL, IADL) and cognitive (MMSE) scales. Acta Neurol Scand 90, 293-298.

[26] Perneczky R, Wagenpfeil S, Komossa K, Grimmer T, Diehl J, Kurz A (2006) Mapping scores onto stages: Mini-mental state examination and clinical dementia rating. Am J Geriatr Psychiatry 14, 139-144.

[27] Jackson C (2011) Multi-State Models for Panel Data: The msm Package for R. J Stat Softw 38, 28.

[28] Jackson C. Multi-state modelling with R: The msm package. The Comprehensive $R$ Archive Network. https://cran.rproject.org/web/packages/msm/vignettes/msmmanual.pdf. Published March 18, 2019. Accessed April 12, 2019.

[29] Bloudek LM, Spackman DE, Veenstra DL, Sullivan SD (2011) CDR state transition probabilities in Alzheimer's disease with and without cholinesterase inhibitor intervention in an observational cohort. J Alzheimers Dis 24, 599607. 
[30] Zhang J, Yu KF (1998) What's the relative risk? A method of correcting the odds ratio in cohort studies of common outcomes. JAMA 280, 1690-1691.

[31] Medicare Provider Utilization and Payment Data: Part D Prescriber. Centers for Medicare \& Medicaid Services Web site. https://www.cms.gov/Research-Statistics-Dataand-Systems/Statistics-Trends-and-Reports/MedicareProvider-Charge-Data/Part-D-Prescriber.html. Updated May 15, 2018. Accessed February 28, 2019.

[32] (2015) Active ingredient: Rivastigmine. RED BOOK Online. Micromedex Healthcare Series [database online]. Truven Health Analytics, Greenwood Village, CO. Accessed December 17, 2020.

[33] Drummond MF, Sculpher MJ, Claxton K, Stoddart GL, Torrance GW (2015) Methods for the Economic Evaluation of Health Care Programmes, OUP Oxford.

[34] Neumann PJ, Cohen JT, Weinstein MC (2014) Updating cost-effectiveness - the curious resilience of the $\$ 50,000$ per-QALY threshold. $N$ Engl J Med 371, 796-797.

[35] Dashputre AA, Kamal KM, Pawar G (2017) Costeffectiveness of peginterferon beta-1a and alemtuzumab in relapsing-remitting multiple sclerosis. J Manag Care Spec Pharm 23, 666-676.

[36] Briggs AH (2000) Handling uncertainty in cost-effectiveness models. Pharmacoeconomics 17, 479-500.

[37] Briggs AH, Claxton K, Sculpher MJ (2006) Decision Modelling for Health Economic Evaluation, Oxford University Press.

[38] Briggs AH, Weinstein MC, Fenwick EAL, Karnon J, Sculpher MJ, Paltiel AD (2012) Model parameter estimation and uncertainty: A report of the ISPOR-SMDM Modeling Good Research Practices Task Force-6. Value Health 15, 835-842.

[39] Caro JJ, Nord E, Siebert U, McGuire A, McGregor M, Henry D, de Pouvourville G, Atella V, Kolominsky-Rabas P (2010) The efficiency frontier approach to economic evaluation of health-care interventions. Health Econ 19, 1117-1127.

[40] Knapp M, King D, Romeo R, Adams J, Baldwin A, Ballard C, Banerjee S, Barber R, Bentham P, Brown RG, Burns
A, Dening T, Findlay D, Holmes C, Johnson T, Jones R, Katona C, Lindesay J, Macharouthu A, McKeith I, McShane R, O’Brien JT, Phillips PPJ, Sheehan B, Howard R (2017) Cost-effectiveness of donepezil and memantine in moderate to severe Alzheimer's disease (the DOMINO-AD trial). Int J Geriatr Psychiatry 32, 1205-1216.

[41] Howard R, McShane R, Lindesay J, Ritchie C, Baldwin A, Barber R, Burns A, Dening T, Findlay D, Holmes C, Hughes A, Jacoby R, Jones R, Jones R, McKeith I, Macharouthu A, O'Brien J, Passmore P, Sheehan B, Juszczak E, Katona C, Hills R, Knapp M, Ballard C, Brown R, Banerjee S, Onions C, Griffin M, Adams J, Gray R, Johnson T, Bentham P, Phillips P (2012) Donepezil and memantine for moderateto-severe Alzheimer's disease. N Engl J Med 366, 893-903.

[42] Jones R, Sheehan B, Phillips P, Juszczak E, Adams J, Baldwin A, Ballard C, Banerjee S, Barber B, Bentham P, Brown R, Burns A, Dening T, Findlay D, Gray R, Griffin M, Holmes C, Hughes A, Jacoby R, Johnson T, Jones R, Knapp M, Lindesay J, McKeith I, McShane R, Macharouthu A, O'Brien J, Onions C, Passmore P, Raftery J, Ritchie C, Howard R (2009) DOMINO-AD protocol: Donepezil and memantine in moderate to severe Alzheimer's disease - a multicentre RCT. Trials 10, 57.

[43] Bond M, Rogers G, Peters J, Anderson R, Hoyle M, Miners A, Moxham T, Davis S, Thokala P, Wailoo A, Jeffreys M, Hyde C (2012) The effectiveness and cost-effectiveness of donepezil, galantamine, rivastigmine and memantine for the treatment of Alzheimer's disease (review of Technology Appraisal No. 111): A systematic review and economic model. Health Technol Assess 16, 1-470.

[44] Claxton K (1999) The irrelevance of inference: A decisionmaking approach to the stochastic evaluation of health care technologies. J Health Econ 18, 341-364.

[45] Krolak-Salmon P, Dubois B, Sellal F, DelabrousseMayoux JP, Vandel P, Amieva H, Jeandel C, Andrieu S, Perret-Liaudet A (2018) France will no more reimburse available symptomatic drugs against Alzheimer's disease. J Alzheimers Dis 66, 425-427. 